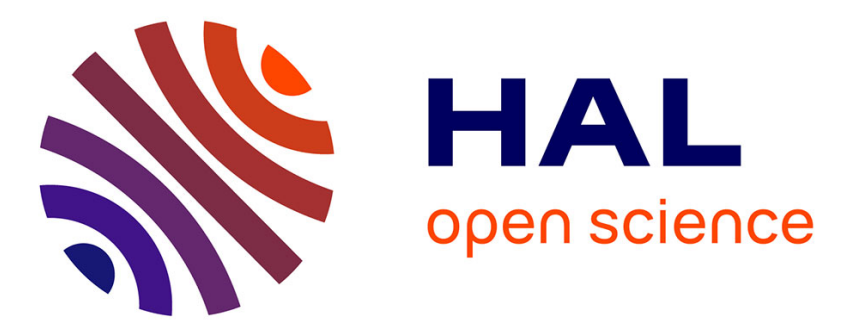

\title{
Téphrostratigraphie et préhistoire des 160 derniers millénaires en Limagne d'Auvergne (Massif Central, France)
}

Jean-Paul Raynal, Gérard Vernet, Serge Sanzelle, Jean Fain, Didier Miallier, Michèle Montret, Thierry Pilleyre, Jean-Pierre Daugas

\section{To cite this version:}

Jean-Paul Raynal, Gérard Vernet, Serge Sanzelle, Jean Fain, Didier Miallier, et al.. Téphrostratigraphie et préhistoire des 160 derniers millénaires en Limagne d'Auvergne (Massif Central, France). Bulletin de la Société préhistorique française, 1994, 91 (2), pp.149-157. halshs-00004137

\section{HAL Id: halshs-00004137 https://shs.hal.science/halshs-00004137}

Submitted on 15 Jul 2005

HAL is a multi-disciplinary open access archive for the deposit and dissemination of scientific research documents, whether they are published or not. The documents may come from teaching and research institutions in France or abroad, or from public or private research centers.
L'archive ouverte pluridisciplinaire HAL, est destinée au dépôt et à la diffusion de documents scientifiques de niveau recherche, publiés ou non, émanant des établissements d'enseignement et de recherche français ou étrangers, des laboratoires publics ou privés. 


\section{TÉPHROSTRATIGRAPHIE ET PRÉHISTOIRE DES 160 DERNIERS MILLÉNAIRES EN LIMAGNE D'AUVERGNE (Massif Central, France)}

Jean-Paul RAYNAL, Gérard VERNET, Jean FAIN, Didier MIALLIER, Michèle MONTRET, Thierry PILLEYRE, Serge SANZELLE, Jean-Pierre DAUGAS

\section{RÉSUMÉ}

Une téphrostratigraphie des retombées cendreuses en Limagne est établie à partir d'affleurements et du sondage profond Géocler 1 dans le maar de Clermont. Elle est étayée par des calages naturalistes et physiques (radiocarbone, thermoluminescence). Entre 160 et $40 \mathrm{ka} \mathrm{BP}$, cent onze retombées (chiffre minimum) caractérisent le volcanisme de la bordure de Limagne et la Chaine des Puys ancienne qui sont bien diftérenciés minéralogiquement. Pendant cette période, huit apports ponctuels de minéraux aciculaires témoignent d'une activité acide lointaine qui reste à localiser. Entre 15 et 7 ka BP, la Chaîne des Puys récente est très active : dans le secteur étudiè, les tephra du Puy de La Nugère du Puy de Dôme, du Puy Chopine, du Cratère Kilian et du Puy de Pariou contribuent largement à la téphrostratigraphie locale. Pour chacune de ces périodes, les implications éventuelles de l'activité éruptive sur la distribution du peuplement préhistorique sont discutees. En conclusion, les perspectives de développement de la recherche préhistorique en zone volcanique sont esquissées.

\section{ABSTRACT}

The Geocler 1 core extracted from the Clermont crater-lake sediments and surface studies made in several localities north of Clermont provided numerous stratigraphic, environmental and chronological data (TL and radiocarbon) which allow us to present a chronostratigraphy of the Limagne for the past $160 \mathrm{ky}$. The Clermont maar eruption has previously been $\mathrm{TL}$ dated to around $160 \mathrm{ky}$. In the crater-lake sediments, between 86 and $28 \mathrm{~m}$ deep, 111 well identified ash-falls have been encountered. No acid tephra has been discovered, but several layers rich in acicular minerals Indicate acid erupliuns, the origin of which remains unclear. Between 15 and $7 \mathrm{ky}, 14$ main ash-falis have been recognized and for some of thom the volcano has been identified: Puy de La Nugère, Puy de Dôme, Puy Chopine, Kilian Crater and Puy de Pariou contribute greatly to the local tephra succession. Perspectives for an increase in prehistoric studies of volcanic areas are outlined.

\section{INTRODUCTION}

Les paléoclimats pléistocènes ont très largement conditionné la distribution des installations préhistoriques et fixé des rythmes d'exploitation du biotope montagnard du Massif Central. Toutefois, le volcanisme actif à plusieurs reprises et en plusieurs points de cette région naturelle eut un impact non négligeable sur los mésoclimats et très certainement sur les comportements humains : l'observation d'une éruption volcanique ne peut laisser indifférent !

Nous avons abordé à plusieurs re prises l'étude de cet aspect particulier des relations homme-milieu aux temps préhistoriques (Raynal et Daugas, 1979, 1984, 1989, 1991 : Daugas et Raynal, 1989, 1991 a et b Raynal et Sanzelle, 1989) et proposons aujourd'hui, à la lumière des travaux récents menés en Limagne d'Auvergne, de faire le point sur quelques caractères de l'activité volcanique et des peuplements humains préhistoriques de ce secteur géographique au cours des cent soixante derniers millénaires.

Rappelons que les téphra sont des retombees volcaniques cendreuses.

\section{DYNAMIQUE}

\section{DES ESPACES NATURELS}

L'organisation structurale du Massif Central détermine son hydrographie et conditionne très largement la distribution des peuplements. L'ossature cristalline du vieux socle a été compartimentée par la lectunique et remodelée par le volcanisme. L'altitude $y$ varie de 300 à 1800 mètres : les hauts plateaux du Velay, du Cantal et du Puy-de-Dôme forment un véritable domaine montagnard, entre 800 et 1200 mètres, coupé de profondes vallées (Loire, Allier) et de bassins tectoniques. Soumise à de fortes influences atlantiques, sa partie occidentale reçoit des précipitations atlantiques importantes d'hiver ct de printemps qui déterminent un cnneigement important. Ces caractères océaniques s'estompent vers l'Est ot les massifs du Sud-Est, quant à cux, sont marqués par un climat nettement plus méditerranéen.

Au cours de la dernière période froide, les hautes terres formèrent une barrière de neige et de glace à l'Ouest ef au Sud (Cantal, Aubrac, Margeride), au Sud-Est et à l'Est (Mézenc, Forez) (Veyret, 1978) : les larges couloirs alluviaux nord-sud de la Loire et de l'Allier furent par conséquent des itinéraires potentiels privilégiés de déplacement des populations animales et humaines dans un domaine ingrat, aux traits physiques et climatiques accusés, exerçant un rôle contraignant sur l'organisation et l'activité des groupes humains.

La dernière phase de déglaciation des zones d'altitude ne nous est pas connue avec précision et l'on hésite encore entre déglaciation précoce (40 ka BP ?) ou tardive (vers 1516 ka BP) (Etlicher et Goër De Hervé, 1988). Des remaniements géomorphologiques importants interviennent après le dernier maximum glaciaire. En Grande Limagne, on observe un surcreusement du cours de l'Allier de 6 à $7 \mathrm{~m}$ au Bölling, puis de plus de $15 \mathrm{~m}$ pendant l'Alleröd et au début du Dryas III (Raynal, 1984). Le régime torrentiel des cours d'eau imposa alors des passages à gué en périodes de basses eaux, facilités 
par les ressauts structuraux qui jalonnent le cours et sont toujours visibles aujourd'hui.

Le volcanisme a perturbé à plusieurs reprises les rythmes geodynamiques à commande climatique et provoqué de profondes transformations de l'envlronnement. Les ecoulements boueux et les laves ont ennoyé le réseau hydrographique : une reprise rapide d'érosion aux flancs des coulées (Pelletier, 1964 ; Blais et al., 1971) s'est alors accompagnée d'une déstabilisation chronique des versants. Certains édifices se sont effondrés et ont provoqué de considérables glissements de terrain (Goër De Hervé el al., 1993). Les projections fines ont été transportées sur de longues distances et ont recouvert de larges territoires modifiant le couvert végétal et l'équitibre géochimique des sols... les paysages ont donc été transformés localement en univers minéraux désertés temporairement par la faune qui avait survécu au cataclysme initial. Des séismes ont accompagné les éruptions et provoqué une modification brutale des versants et des changements du cours des rivières : c'est le cas en Grande Limagne. L'accumulation puis le dégagement de gaz carbonique a certainement génére des pièges mortels. Si l'on admet la concomitance, dans le temps et l'espace, de plusieurs éruptions, les ter ritoires affectes n'ont plus offert pou l'Homme aucun attrait et ce pour plusieurs décennies.

Le relief, les fluctuations climatiques et l'activité volcanique posent donc ici avec une acuité toute particulière les limites de l'interprétation archéologique.

ÉLÉMENTS DE TÉPHROSTRATIGRAPHIE RÉGIONALE

\section{De 160 à 40 ka avant le présent : volcanisme basaltique bordier et naissance de la Chaîne des Puys}

Pour la période considérée, seuls 27 édifices ont été datés directement par TL (Miallier, 1982 ; Guérin, 1983 Raynal et al., 1984 ; Goër de Hervé et al., 1993) et la quasi-totalité entre 100 et $40 \mathrm{ka}$. II convenait de rechercher, au pied du plateau des Dômes,

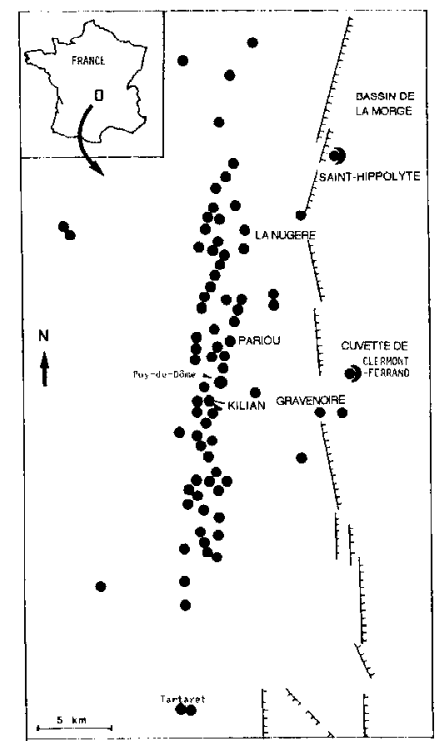

Fig. 1 - Carte schématlque de la Chante des Puys (d'après Camus et al., 1983). Les volcans sont repré-
sentés par des points noirs. Zone d'étude en hasentés par des points noirs. Zone d'étude en ha-
chures ot montion des principales lacalités étu.
diées.

un système enregistreur susceptible de fournir une image plus fidèle de l'activité volcanique régionale. Notre choix s'est porté sur la cuvette de Clermont et sur le bassin de la Morge (fig. 1). Les marqueurs d'éruptions ont été recherchés dans les formations alluviales et les retombées conservées ont été caractérisées par leur cortège minéralogique et leur composition chimique (Vernet, 1992) et datées directement lorsque les conditions le permettaient (Pilleyre, 1991).

La cuvette de Clermont est constitué de plusieurs maars coalescents péné-contemporains installés sur une zone de faiblesse immédiatement à l'Est de la grande faille bordière de Limagne. Les projections de l'un de ces maars, celui de Jaude-Salins, culminent à $410 \mathrm{~m} \mathrm{NGF}$ et constituent la Butte de Clermont (Pelletier, 1969 ; Baudry et Camus, 1970). La première datation par thermoluminescence d'un phénomène phréatomagmatique a été réalisée sur ces pyroclastites (Mlallier 1982 ; Miallier et al., 1983, 1984 ; Raynal et al., 1982, 1985) et situe l'explosion du maar pendant l'avantdernier glaciaire, au cours du stade 6 de la courbe isotopique océanique :

- Cler TL $23=157 \pm 22 \mathrm{ka}$ (Cours Sablon),

- Cler TL $27-156 \pm 22$ ka (Hôtel de Chazerat).

Dans cettc vaste dépression, alimentée à l'Ouest par la Tiretaine, s'est édifić un complexe lacustre reconnu par d'anciens travaux de forage (fig. 2). Le fond de la dépression en zone de cceur de lac n'a pas encore été atteint par sondage, mais les dépôts de cette zone ont cependant été reconnus dans sa partie sud-est ("Fond de Jaude") sur quatre-vingt-six mètres lors du forage Géocler 1 réalisé du 17 novembre au 10 décembre 1986 (Raynal, 1987, 1988). Bien que difficile à évaluer, la puissance totale des dépôts pourrait largement dépasser la centaine de mètres et représenterait donc un bilan d'accumulation des cent soixante derniers millénaires. Des repères chronologiques sont disponibles par la datation directe de certaines tephra par TL (Pilleyre, 1991 : Pilleyre et al., 1991) :

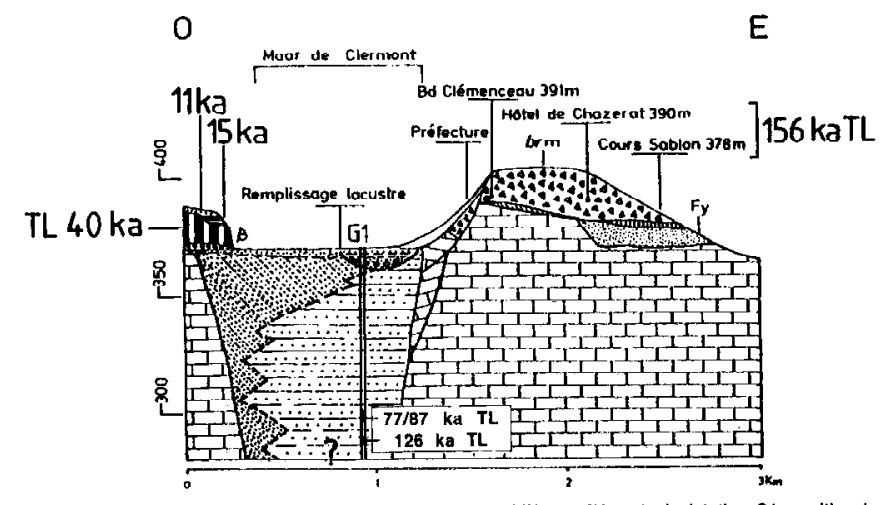

Fig. 2 - Le maar do
forage GEOCLER 1. 
- Cler TL. $250=126 \pm 15 \mathrm{ka}$ à $70 \mathrm{~m}$ de profondeur,

- Cler TL 251 a $=88 \pm 10$ ka à $62 \mathrm{~m}$ (fraction $200315 \mu \mathrm{m}$ ),

- Cler TL 251 b $=77 \pm 9$ ka à $62 \mathrm{~m}$ (fraction 100-200 $\mu \mathrm{m}$ )

Un tel système lacustre, était propice à l'enregistrement d'éruptions volcaniques plus ou moins proches: entre 28 et $86 \mathrm{~m}$ de profondeur, outre l'extrême base de la carotte extrëmement riche en tephra, $118 \mathrm{re}$ tombées bien individualisées dont 111 tranchement directes, sous forme de fines lamines ou passées $(1 \mathrm{~mm}$ à quelques centimètres) de couleur sombre (noir, gris, rouille) et comportant pour certaines des scories ont été considerées comme des apports directs et étudiées. Aucune retombée acide vraie n'a été repérée. mais des apports ponctuels de minéraux aciculaires associés à des scories leucocrates sont interprétés comme l'indice d'eruptions acides bien particulieres dont la localisation n'a pu être établie. Cette étude permet de conclure à l'existence de deux grandes phases volcaniques (fig. 3 et 4)

\section{- PHASE 1}

Enregistrée de 86 à $62 \mathrm{~m} .13$ retombées directes minimum. Le mi néral caractéristique est un clinopy roxène brun automorphe mâclé en croix ; on note des pourcentages plus faibles de pyroxène vert et la quasi-absence de l'olivine; dans les fractions grossières, les tephra contiennent souvent des scories noires bleutées très vésiculées. Cette phase est également enregistrée dans les alluvions du bassin de la Morge et une des retombées a été fossilisée au sein du massif de travertins de Rouzat. Vers la fin de cette période intervient l'éruption de maar de Saint-Hippolyte : le minéral caractéristique en est une olivine automorphe présentant des lacunes de cristallisation. Cette éruption n'a pas été identifiée dans la carotte Géocler 1 mais est enregistrée dans les alluvions du bassin de la Morge Cette phase correspond donc ì un volcanisme basaltique dispersé en bordure de Limagne. D'après les données de chronologie numérique, elle prendrait fin vers $70 \mathrm{ka}$

- PhASe 2

Enregistrée de 62 à $28 \mathrm{~m} .75$ retombées directes minimum. Le minéral ca-

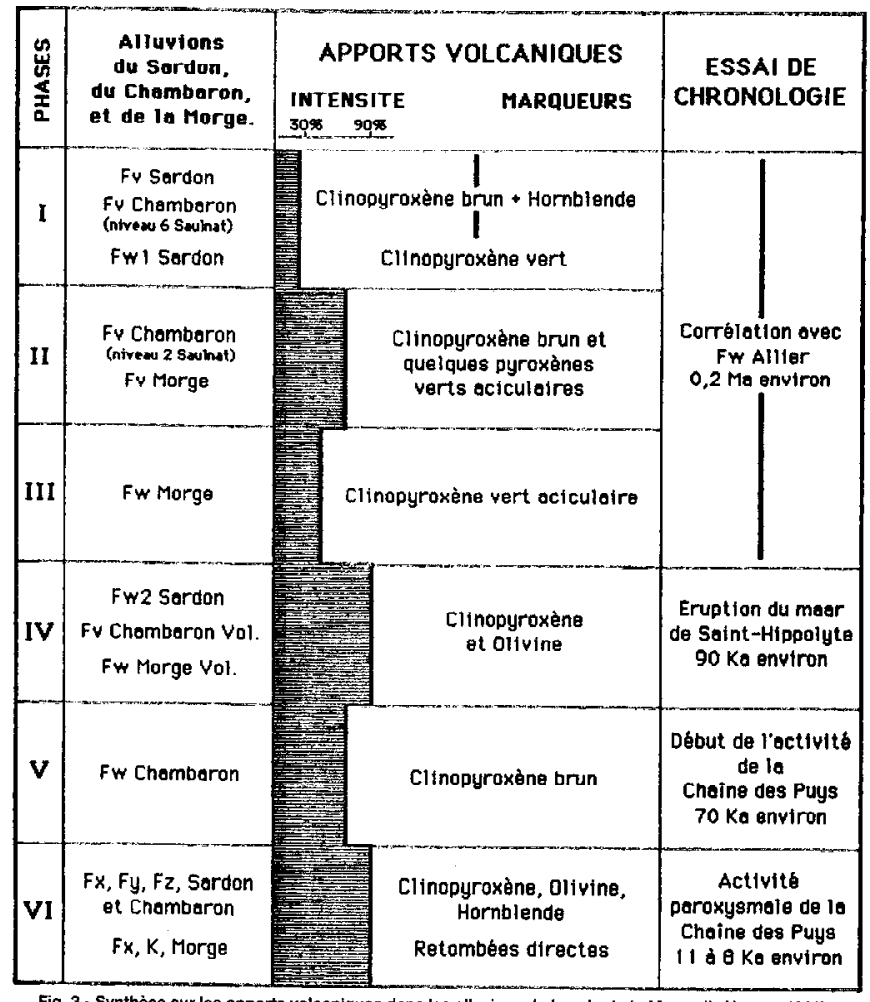

Fig. 3 - Synthèse sur les apports volcaniques dans les alluvions du bassin de la Morge (in Vernet, 1992).

ractéristique est le clinopyroxène vert en éclats ; le clinopyroxène brun est quasi absent; l'olivine est le plus souvent abondante ot individualise des sous-phases. Les scories sont fines. Cette phase est bien marquée dans les alluvions du bassin de la Morge. Elle correspond à la phase ancienne de la Chaîne des Puys qui débuterait vers $70 \mathrm{ka}$

De 160 à 30 ka BP, l'activité volcanique en bordure occidentale de la Limagne est donc continue. Le paysage est en constante modification sous l'effet du volcanisme et l'Homme doit tenir compte de ces manifestations. On nous objectera qu'une éruption par millénaire ne représente pas un contexte apocalyptique de nature à perturber des habitudes ancestrales. Nous répondrons que la fréquence des éruptions établie par les différentes méthodes n'est qu'une estimation minimum et que nous devons nous garder d'un comparatisme hâtif avec les exemples récents de recolonisation rapide du milieu volcanique par l'Homme moderne.

\section{A partir de 15 ka avant le présent : le temps des Dômes}

Pendant le Dryas, l'Alleröd, le PréBoréal, le Boréal puis l'Atlantique, la Basse-Auvergne connaît un volca nisme très actif dans la Chaîne des Puys et à sa périphérie.

Une activité strombolienne est responsable de la construction de nombreux cones, de la projection de tephra basaltiques et de l'émission de longues coulées de laves fluides (Tartaret, Montchal, Montcineyre, La Vache et Lassolas...) qui ennoient les vallées des affluents de rive gauche de l'Allier et recouvrent peut-être des sites magdaléniens (Daugas et Raynal, 1979). S'y ajoutent les explosions phréatomagmatiques de nombreux maars trachytiques (Kilian. Pavin) et trachyandésitiques (Nugère. Pariou) accompagnées de larges projections de tephra provoquant de brutales et radicales modifications des écosystèmes et des éruptions péléennes à l'origine de l'édification de grands ap- 


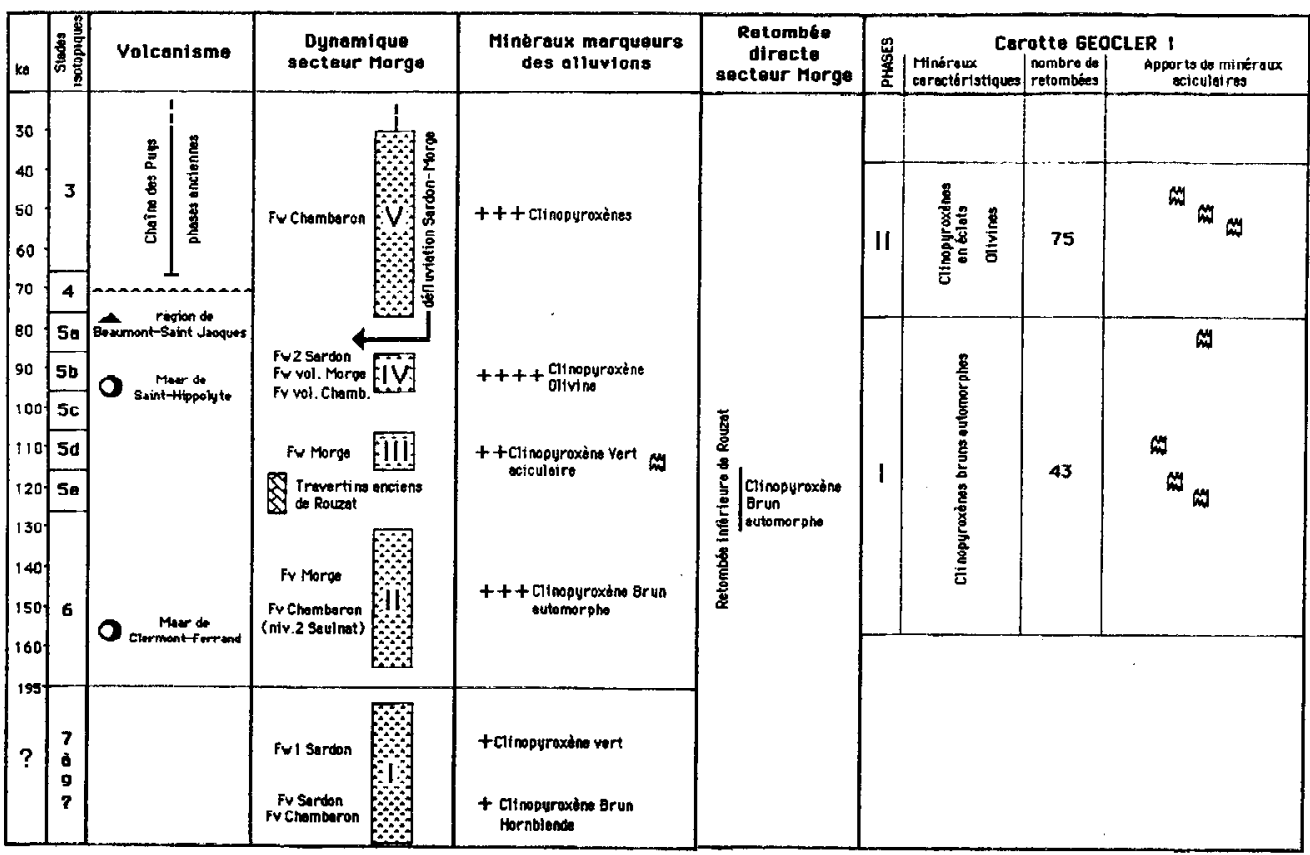

Fig. 4 - Essai de synthèse dynamlque et téphrostratigraphlque en Limagne nord-occidentale de 300 à $30 \mathrm{ka}$ (in Vernet, 1992).

pareils trachytiques (aiguilles et cumulo-dômes du Puy de Döme, du Sarcouy, du Chopine...). Ces eruptions pliniennes, dévastatrices, dont les panaches peuvent atteindre $20 \mathrm{ki}-$ lomètres de hauteur (Kieffer et Camus, 1981), dispersent des produits fins sur de très vastes zones géographiques.

Une revue critique de la documentation montre que peu d'appareils de la Chaîne des Puys sont directement datés :

- Puy de Côme, $15900 \pm 1500$ et $11600 \pm 830$ (Guérin, 1983).

- Puy de Lassolas, $15700 \pm 1700$ (Guérin, 1983)

- Puy de Tartaret, $13700 \pm 1600$ (Pilleyre et al., 1992).

- Puy de Gorce, $13200 \pm 1300$ (Guérin, 1983).

- Puy de Barme, $11900 \pm 1200$ (Guérin, 1983).

- Puy de la Nugère lava flow. $10900 \pm 1200$ (Guérin, 1983).

- Puy de Dôme, $10800 \pm 1100$, $9300 \pm 1100$ (Fain et al., 1986, 1991).
- Puy de Pariou lava flow, $8180 \pm$ 810 (Guérin, 1983).

- Puy de la Vache, $8100 \pm 800$ (Huxlable et al., 1978), $9150 \pm 550$ et $8820 \pm 870$ (Guérin, 1983), 9130 \pm 720 (Montret et al., 1992).

- Puy de Montchal, $7560 \pm$ 770 BP (Guérin, 1983)

Pour apprécier l'importance et fréquence des éruptions pendant la période considérée, il est donc nécessaire de faire appel aux données fournies par les recouvrements de tephra localisés dans les milieux de sédimentation propices à leur conservation, dans la Chaîne et à la périphérie de la zone éruptive. L'apport de nouvelles données téphrostratigraphiques et chronologiques obtenues dans la cuvette de Clermont-Ferrand et en Limagne septentrionale (Pilleyre, 1991 ; Vernet, 1992) complète les informations obtenues en surface et dans les lacs et tourbières (Bastin et al., 1990 . Baudry et Camus, 1970 ; Camus, 1975 ; Guénet, 1986 ; Juvigné, 1987 ; Juvigné et Bastin, 1982 ; Juvigné et Gilot, 1986 ; Juvigné et Gewelt, 1987 ; Marambat, 1986, 1991). Toutefois, l'attribution d'une retombée à un appareil déterminé reste encore déli- cate. L'ordre d'émission des différents recouvrements (et l'identification du volcan source lorsque cela s'avère possible) ayant affecté la Limagne est le suivant, du plus ancien au plus récent (fig. 5) :

- Tephra de Cellule, à composition trachyandésitique, situées par la palynologie dans le Dryas ancien. II pourrait s'agir d'un recouvrement lié à une phase éruptive du Puy de la Nugère (Vernet et Paquereau, 1986, 1991 ; Vernet et al., 1990)

- Tephra des Roches, à composition trachyandésitique, reconnue dans deux stratigraphics situées en Limagne septentrionalc, pout être liées au Puy de la Nugère: datation radiocarbone de $12010 \pm 150 \mathrm{BP}$ (Gif TAN 91102) ; elle a été repéréc en contexte archéologique, au sommet du remplissage du gisement magdalénien d'Enval (Vernet, 1991, 1992).

- Tephra de la Moutade, à composition de basalte andésitique $(\mathrm{K}$ Hawaiite), reconnues en différents points de la Grande Limagne septentrionale et peut-être liées au Puy de la Nugère : datation directe par thermoluminescence de $13700 \pm$ 1700 (Cler TL 110) (Vernet et al. 1990 ), calage palynologique dans 
l'Alleröd et datation radiocarbone de $11360 \pm 130$ BP (Ly 3733) Nernet et Paquereau, 1986, 1991).

- Tephra du maar trachyandésitique de la Nugère comprises entre $11490 \pm 60 \mathrm{BP}(\mathrm{GrN} 12643)$ et $11340 \pm 100$ B.P. (GrN 12 642) (Etlicher et al., 1987).

- Tephra supérieure de Gi meaux, à composition trachyandésitique (K-Benmoréite), d'âge tardiglaciaire sensu lato. Le volcan source n'est pas connu pour l'instant, mais il pourrait s'agir d'une phase ćruptive du Puy de la Nugère (Vernet, 1991).

- Nuées du Puy de Dôme : des produits trachytiques fins expulsés dans l'atmosphère ont été transportés par des vents dominants d'Ouest et répartis sur les régions du NordEst (Juvigné et Bastin, 1982), de l'Est et du Sud-Est de la France et jusqu'en Suisse (Martini, 1970), pendant le Pré-Boréal ; les dates récemment obtenues directement sur cet appareil (Cler TL 90-A : $10800 \pm 1000$ et Cler TL 90-C : $9800 \pm 1000$ ) (Fain et al., 1986) appuient cette proposition.

- Tephra supérieures de Rouzat, à composition de basalte andésitique (K-Hawaiite), peuvent être situées, par leur position stratigraphique au sien d'un massif travertineux, dans le début de l'Holocène. Le volcan source n'est pas connu pour l'instant (Nernet, 1992).

- Nuées trachytiques de type "Coquilie" (Carrus, 1975) rapportées au Puy Chopine : $8900 \pm 190 \mathrm{BP}$, $8410 \pm 150$ BP (Gif 2114), $8200 \pm$ $300 \mathrm{BP}$ (Gif 1501), $8150 \pm 150 \mathrm{BP}$ (Gif 2113) ; $8760 \pm 170 \mathrm{BP}$ (Gif 3638), $8610 \pm 160$ BP (Gif 5 254) (Raynal et al., 1981) ; $8970 \pm 115 \mathrm{BP}$ (MBN 343) (Juvigné et Gewelt, 1987).

- Tephra CF1 lou retombée du Creux-Rouge), à composition de KMugéarite, sont présentes dans plusieurs stratigraphies de la cuvette de Clermont. L'âge de la mise en place de cette retombée est imprécis mais compris entre la fin du Tardiglaciaire et le Boréal ( Raynal et al., 1979).

- Tephra CF2 lou retombée de la rue Descartes), à composition de K-Hawaiite, sont datées directement par TL à $8700 \pm 900$ avant 1980 (Cler 114) (Raynal et al., 1989) et calées par la palynologie sur une autre coupe de la cuvette de Clermont

$\mathbf{A}$

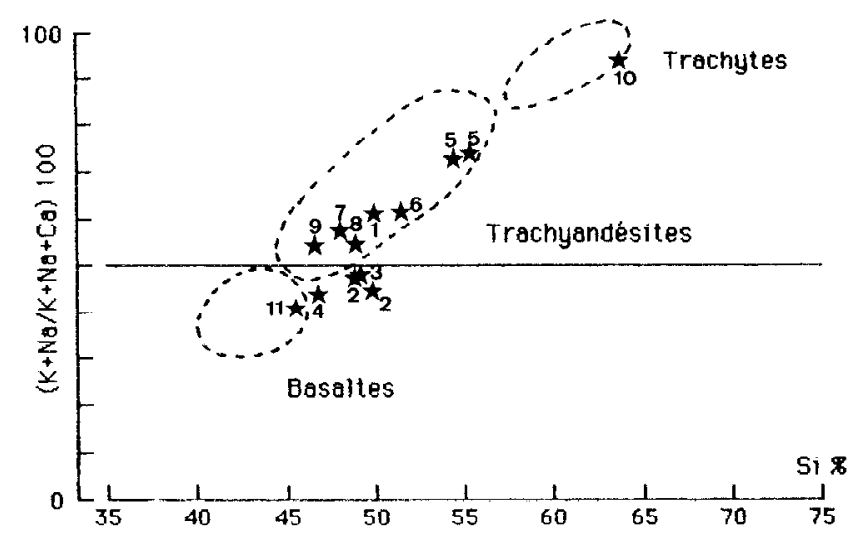

B

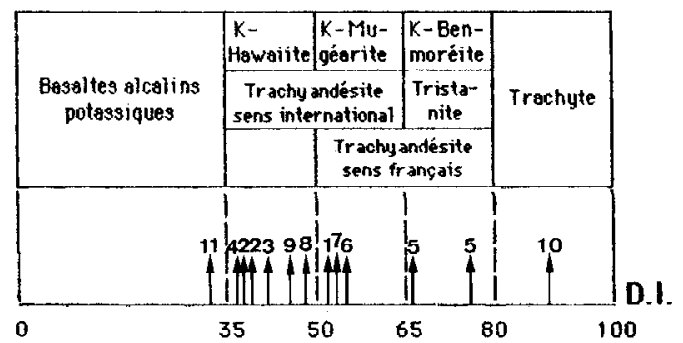

Fig. 5 - Composition chimique des différentes tephra étudiées reportées sur le diagramme Si/R de Brousse (1971) et dans la classification de Maury suivant le Dl. 1 : tephra de Cellule. 2 : tephra de La Moutade (2 samples).

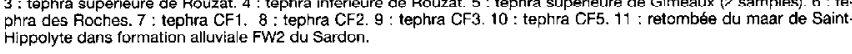

entre 9 oUU et $8000 \mathrm{BP}$ (fin du Boréal/Boréal).

- Tephra CF3 lou retombée de la rue de Montjuzet), à composition de K-Hawaiite, sont présentes dans toutes les stratigraphies étudiées dans la cuvette de Clermont. Elles présentent un faciès très particulier (fig. 6) : à la base un mince niveau rougeâtre caractéristique d'un effet de "blast" phréatomagmatique, puis un mélange de produits caractéristiques d'une éruption mixte (magmatique et phréatomagmatique), enfin des scories vésiculaires caractéristiques d'une retombée de panache strombolien. L'origine de ces tephra, calées par la palynologie entre 9000 et $8000 \mathrm{BP}$, reste pour l'instant incertaine (Vernet, 1992).

- Tephra CF4 (ou retombée ancienne de la rue de Blanzat), à composition de K-Hawaiite, possè- dent souvent un caractère phréatomagmatique accusé et se trouvent situées dans le même Intervalle chronologique que les précédentes: fin du Préboréal/Boreal. L'origine est incertaine mals très probablement identlque à celle de CF3 (Vernet, 1992).

- Tephra CF5 (ou retombée de la rue Sous-les-Vignes), à composition de trachyte, sont présentes dans une seule des stratigraphies de la cuvette de Clermont ou elles sont calées palynologiquement à la fin $d u$ Boréal/début de l'Atlantique. L'âge de cette retombée trachytique - la première identifiée en Limagne - et sa composition minéralogique (l'absence de Sphène exclut le Puy Chopine ; Bentor, 1955) nous permettent de proposer comme volcan source le Cratère Kilian (Vernet, 1992). Une très vaste dispersion a récemment été proposée pour des tephra tra- 


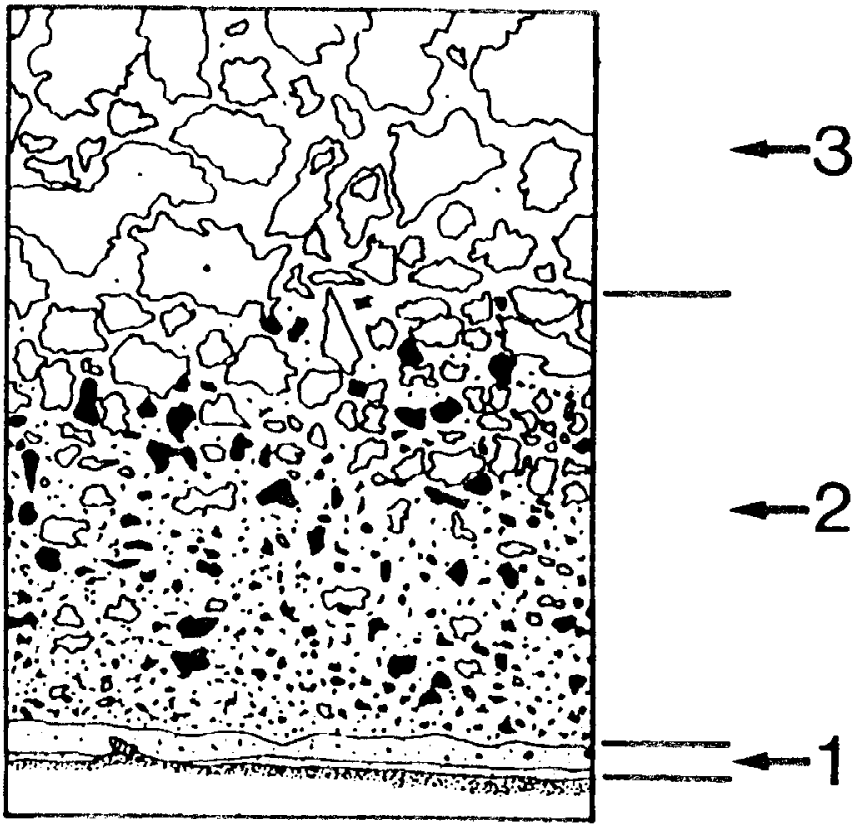

Fig. 6 -

chytiques de la Chaîne des Puys (Vasset ou Kilian) d'âge Boréal (Juvigné, 1991).

- Tephra CF6 lou retombée récente de la rue de Blanzat), à composition trachyandésitique, observées dans une coupe de la cuvette de Clermont, pourraient bien être le témoin d'une phase éruptive du Puy de Pariou (Vernet, 1992).

It s'y ajoute, dans l'extrême Sud de la région considérée, les retombées suivantes:

- Tephra de Montcineyre : 6000 BP (Guénet, 1986) ; $6530 \pm$ $100 \mathrm{BP}$ (LV 1522), $6520 \pm 100 \mathrm{BP}$ (Lv 1523) (Juvigné et Gilot, 1986).

- Tephra du maar trachytique du Pavin : 5800 BP (Guénet, 1986 : Marambat, 1986) ; $5680 \pm 100 \mathrm{BP}$ (Lv 1491), 5 990 80 BP (Lv 1492), $5710 \pm 90 \mathrm{BP}$ (Lv 1493) (Juviané et Gilot, 1986) ; $5990 \pm 140$ BP (MBN 327) (Juvigné et Gewelt, 1987).

II convient de calibrer les dates radiocarbone (signalées en BP) afin de les comparer avec les dates obtenues par thermoluminescence qui sont données en années réelles avant aujourd'hui. On observe alors, malgré de beaucoup plus larges incertitudes statistiques, un resen années réelles. des quelques gisements stratifiés ne paraissent pas standardisés et semblent surtout répondre aux opportunités mésologiques. Aussi nous bornerons-nous à constater que :

les traces d'Acheuléen sont plus fugaces en Grande Limagne (rares bifaces isolés) qu'au Nord (Bourbonnais) ct au Sud (haute vallée de l'Allier à partir de Brioude et Velay),

- les occupations moustériennes sont anciennes (100 à $80 \mathrm{ka}$ ) tant sur la bordure occidentale de la Limagne qu'en Velay (Raynal et Huxtable, 1988 ) et les rives de lacs de cratères et les plages alluviales (ressources en eau et ressources animales) sont occupés temporairement (Saint-Hippolyte, Les Mayes) (Raynal et al., 1984 ; Vernet, 1992). Pendant les phases éruptives, les itinéraires de pénétration du massif empruntent peut-être les vallées de la Dore et/ou de la Loire pour gagner le Velay où l'absence de matière première lithique originaire du Sud-Est pourrait être une conséquence de l'activité volcanique en Vivarais (Raynal et Daugas, 1984). serrement des périodes éruptives entre 13000 et 7000 avant le présent

On retiendra l'opposition entre la concentration géographique du volcanisme tardiglaciaire et holocène de Basse-Auvergne et la partois très large dispersion de ses produits, bien au-delà des frontières régionales et on notera son interêt chronostratigra phique pour une periode particulièrement importante de la Préhistoire.

\section{OCCUPATIONS DE L'ESPACE RÉGIONAI}

Pour la période 160 à $40 \mathrm{ka}$, le bilan de nos connaissances reste très pauvre. L'activité explosive du massif des Dores jusque vers $0,2 \mathrm{Ma}$ est relayée par celle du volcanisme bordier de Limagne, puis par la Chaine des Puys ancienne à partir de 70 ka BP environ. Pendant l'avantdernier glaciaire (stade isotopique 6), les conditions idéales de peuplement (pause de l'activité volcanique et amélioration climatique synchrone) ne sont donc pas réunies mais le nombre relativement réduit de gisements et leur grande dispersion chronologique interdit toute ap proche détaillée. Plus, les comportements suggérés par l'analyse
De 40 à 25 ka, les épigones du Moustérien el les prernières cultures du Paléolithique supérieur (Castelperronien, Aurignacien) ne paraissent pas avoir pénétré le massir : conséquence de l'activité volcanique de la Chaîne des Puys et de l'instauration progressive des conditions pléniglaciaires ou suite logique du remplacemerıl de population et des modifications des modes de subsistance?

A partir de 25 ka BP, les populations du Périgordien final et du Protomagdalénien gagnent les hautes vallées de la Loire et de l'Allier à la faveur de l'amélioration climatique de Tursac mais leurs traces n'ont pas été décelées en Basse-Auvergne...

Entre 19 et 15 ka BP, alors que se fait jour une recrudescence du volcanisme en Basse-Auvergne et à la faveur d'une rémission climatique de faible amplitude (interstade de Lascaux) les groupes du Magdalénien ancien atteignent le Velay, tant par le Val d'Allier (Creuzier / $260 \mathrm{~m}$, Le Sire / 350 m, Le Blot / 500 m, La Roche à Tavernat $/ 580 \mathrm{~m}$ ) que par le cours de la Loire (Cottier / $600 \mathrm{~m}$, Le Rond du Barry / $850 \mathrm{~m}$ ). Cependant, l'absence de site important du Magdalénien ancien en Basse-Auvergne s'explique peut-être par une recrudescence de l'activité volcanique: les cendres volcaniques riches en 
fluorine qui avaient saupoudré la région blessaient le palais des herbivores, les empêchant ainsi de se nourrir, contraignant les hardes à choisir temporairement d'autres itinéraires de migration (vallée de la Dore et de la Loire) et forçant l'Homme à faire de même. Cette perturbation des itinéraires traditionnels se marque dans l'approvisionnement en matériaux lithiques.

Après 15 ka, commence la dernière phase de construction de la Chaîne des Puys. Le peuplement du Magdalénien supérieur est alors sans doute fondé sur une exploitation tournante rationnelle des biotopes, rythmée par le cycle du Renne: migration en fin de saison froide vers les collines granitiques de HauteAuvergne et du Velay pour la mise bas, descente en fin d'été vers les espaces d'hivernage. Le Renne n'aimant pas les plaines larges mais les vallons ramifiés et contournés, évitant les étendues de marais et les zones saupoudrées par le volcanisme, les voies de passage le long de l'Allier suivaient les bords de la Limagne. L'Homme suivait ce déplacement. utilisant les gués et les passages resserrés pour chasser cette espèce au printemps et à l'automne : on connaît des sites d'abattage (Creuzier, Blanzat) placés au débouché de vallons ouvrant vers les collines du Forez et des Combrailles, ou occupant des positions stratégiques sur la vallée de la Loire (Goutte-Roffat. Rocher de la Caille). Pendant l'estive, l'Homme exploitait les zones d'altitude à partir de camps de base proches des concentrations de rennes (Le Rond du Barry. Tatevin...), chassant le Cheval et le Bouquetin et pratiquant la trappe (marmottes) et la pêche. Ces activités de substitution estivales prêtaient sans doute plus à consommation directe de viande et exploitation des matières animales qu'à préparation de viande pour stockage (problème de transport), bien que le doute subsiste (faute de document) à propos d'une éventuelle pêche extensive au gué des salmonidés en Val d'Allier, autorisant le stockage d'une ressource saisonnière abondante (Le Gall, 1988 et com. pers.). Dans cette perspective, chaque site trouve sa place et les vides des cartes de répartition ont une explication logique : territoires englacés de haute altitude, terroirs au substrat calcaire délaissés autant que possible par le Renne, étendues de Basse-Auvergne affectées par les retombées volcaniques, désaffection des zones pa- lustres de Limagne (prolifération esti vale des moustiques, déformations par l'engel et le thermokarstl et négligence des bords de lacs situés hors du trajet du Renne (en zone volcanique) ou trop en altitude pour attirer les espèces grégaires.

Le regain de l'actlvité volcanique a-t-il perturbé ces comportements ? Les retombees identifiees dans le gisement d'Enval, rapportees à une phase eruptive du Puy de la Nugère vers $12 \mathrm{ka} \mathrm{BP}$, scellent le dernier niveau d'occupation et pourraient bien être la cause de l'abandon de ce site-sanctuaire et à l"origine de modifications irréversibles des habiludes ancestrales!

Avec la fin du Paléolithique, la désaffection pour la Basse-Auvergne ira croissant et se maintiendra pendant près de trois millénaires, jusqu'à la fin du Mésolithique. L'activité paroxysmale de la Chaîne des Puys maintient en effet en Basse-Auvergne des conditions particulièrement défavorables à la présence de gibier et de ressources végétales spontanées : les Dômes ajoutent au paysage la touche finale que nous lui connaissons. Les activités des chasseurs de l'Épipaléolithique, puis du Mésolithique, se déplacent vers les hauts plateaux et les massifs maintenant désenglacés où la progression sylvatique rapide génère des espaces giboyeux : aucun site mésolithique n'a été jusqu'à ce jour repéré en Basse-Auvergne.

\section{"RETOMBÉES" \\ CONCLUSIVES}

Au terme de cette étude, les apports du sondage Géociler 1 et des études microrégionales entreprises en Limagne septentrionale complètent et précisent singulièrement l'image de l'activité éruptive de la Chaîne des Puys et des massifs environnants. L'idée que nous pouvons nous faire des rapports Homme-Volcan pendant la Préhistoire reste encore très floue, même si certains instantanés sont particulièrement révélateurs.

Cette approche permet en tout cas de cerner des objectifs à moyen terme pour l'Archéologie préhistorique des zones volcaniques et particulièrement la Basse-Auvergne. Elle ne peut se concevoir en dehors d'un cadre pluridisciplinaire et repose largement sur l'établissement d'une téphrostratigraphie régionale raisonnée. Ceci suppose:

- le renouveau des études microrégionales en matière de géologie du Quaternaire, la réalisation plus systématique de sondages carottés dans des structures d'accumulation lente judicieusement choisies (paléo-lacs de maars ou de barrage, tourbières) le recours intensif à la datation directe (thermoluminescence) et indirecte (radiocarbone, palynologie) des tephra pour l'établissement d'une correspondance entre calendriers au-delà de la limite actuelle de calibration, l'analyse minéralogique détaillée et systématique des dépôts archéologiques pour y repérer les isochrones que sont les recouvrements téphriques

- un réexamen des sites archéologiques qui implique (liste non limitative) leur datation précise par des approches croisées, l'étude détaillée et sur des échantillons représentatifs des saisons d'abattage de chaque espèce animale, la caractérisation de l'environnement végétal de chaque occupation, l'identification par des techniques appropriées de la fonction des outillages lithiques, l'étude approfondie des matières premières lithiques et la définition de leur sens de circulation, la comparaison de ces derniers à ceux des objets de "collection", l'établissement des chaînes opératoires de la fabrication des objets en matières dures animales, la caractérisation de l'art mobilier et de ses relations stylistiques avec les aires géographiques environnantes...

.. Autant de clés pour une meilleure évaluation de l'impact des éruptions sur les comportements humains et donc une meilleure compréhension de l'organisation régionale des stratégies d'exploitation des terroirs volcaniques.

\section{Remerciements}

Ce travail a bénéficié des soutiens de l'A.T.P. "Transfert de technologie" de l'IN2P3, programme Datation par thermoluminescence et volcanisme quaternaire de la Chaine des Puys, coordination J. Fain, de l'A.T.P. "Approches nouvelles en Archéologie par le biais des Mathématiques, de la Physique, de la Chimie et des Sciences de la Terre" du C.N.R.S. programme Peuplement préhistorique en zone volcanique active coordination J.-P. Raynal, et du concours de la Direction des Antiquités Préhistoriques d'Auvergne. 


\section{Références}

Basiln B., GeWELI M. el Juviginé E. (1990) - A propos de l'âge et de l'origine des téphras tardiglaciaires T4 et T5 de Godivelle-nord (Massif Central, France). Ann. Soc. Géol. Belg., tome 113 (fasc. 2), p. 165-178, 7 fig., 1 tabl., 1 pl.

Baudry D. et Camus G. (1972) - Les pro jections volcaniques de la Chaine des Puys et leurs utilisation
B.R.G.M., (2), 11,2, p. $1-52$

BeNtoR Y.K. (1955) - La chaine des Puys (Massif Central français) : recherches géologiques et petrographiques. Bull. du Service de la Carte Géologique de la France, n०242, tome LII, 1954 787 p., fig. 75 , tabl. CLXX.

Blais S., Maury R. et Brousse R. (1971) - Contribution à l'estimation des vitesses de creusement des cours d'eau au flanc de coulées basaltiques. Travaux de I'Institut de Géographle, XLIII, Faculté des Lettres de Clcrmont For rand, p. 3-15

Brousse R. (1971) - Magmatologie du volcanisme néogène et quaternaire $d u$ Massif Contral. In Jung J. symposium : Géologie, géomorphologie et structure profonde du Massif central français. Plein Air Service Éd., Clermont-Ferrand, p. 377-478.

Cavus G. (1975) - La Chaine des Puys Atude structurale et volcanologique. Annales Scientifiques de l'Universite de Clemont, $n 56$, Géol. Minéral. fasc. 28 , fig. $68,322 p$.

DaUGAS J.-P. et RAYNAL J.-P. (1979) - Remarques sur le milieu physique et peuplement humain en Auvergne à la fin des temps glaciaires. In La fin des temps glaciaires en Europe, C.N.R.S
Éd., Paris (1979), t. 2, p. 545-562.

Daugas J.-P. et RaYnal J.-P. (1983) - Paléomilieux et comportements humain de la fin des temps glaciaires a l'Atlantique dans le Sud du Massif Central. I Premières communautés paysanne en Méditerranee occidentale, coll. intern. C.N.R.S. Montpellier (1987) Paris, G.N.R.S. Ėd., p. 107-124.

DAUGas J.-P. et Raynal J.-P. (1989) Quelques étapes du pcuplcment du Massif Central francais dans leur contexte paleoclimalique et paleogeographique. In Laville $H$. : Variations de palémilleux et peuplement préhistorique, C.N.R.S. Éd., Cahicrs du Qua ternaire, $1989, n^{\circ} 13$, p. 67-95.

DaUGas J.-P. et RaYNal J.-P. (1991 a) L'homme et les volcans : mésolithisation et neolithisation dans le Massif Central français, $113^{\circ}$ Congr. nat. Soc. et Néolithisation, p. 127-146, 3 fig. 3 labl.

DaUgas J.-P. et RAYNaL J.-P. (1991 b) Les chasseurs de la préhistoire face aux dangers du volcanisme. La depêche du parc, Bull. d'information du parc naturel régional des volcans d'Auvergne supolément scientifique. $n^{\circ} 5$, juin 1991 , p. $4-5$.
Escalon de fonton M. et Brousse R. (1969) - Currélaliun entre les phases d'effondrement dans les grottes préhistoriques et les phases d'activité historiques et les phases d'activite France. XIX session, Auvergne, Paris, S.P.F. (1972), p. 200-223.

Etulcher B., Janssen C., Juvigne E. et Van LEEUWEN J F N (1987) - Le Haut LEEUWEN J.F.N. (1987) - Le Haut Forez (Massit Central, France) apres le pleniglaciaire wurmien : environgère. Bulletin de l'Association trangère. Bulleth de lassociation trançaise pour letude
p. $229-239$.

Fain J., Erramli H., Mialuer D., Montret $M$. et SANZELLE S. $(1986)$ - Datation par thermoluminescence d'un appareil volcanique trachytique : le Puy de Dôme. $11^{*}$ R.S.T., Clermont-Ferrand,

Fain J., Erramli H., Miallier D., Montret $M$. et SANZELLE $S$. (1991) - Datation par thermoluminescence d'un appareil volcanique trachytique: le Puy de Dóme. In J.-P. Raynal et D. Miallier : Datation et Caractérisation des Milieux Pleistocenes, Cahlers du Quaternaire, C.N.R.S. Ed., n 16, p. 53-6?

GUÉRIN G. (1983) - Thermoluminescence des plagloclases. Méthode de datation du volcanismo. Applications au domaine volcanique français: Chaîne des Puys, Mont Dore et Cézallier, Bas-Vi-
varais. Thèse d'État Université Pierrevarais. Thèse d'Etat, Universite
et-Marie-Curie, Paris, $253 p$.

GuÉNET P. (1986) - Analyse pollinique de la tourbière de Chambedaze et recherches pollenanalytiques dans les Monts Dore et le Cézallier, Massir Central, France. Thèse de l'Université d'Aix-Marseille III.

Etlicher B, et Goér de Hervé A. de (1988) - La déglaciation würrnienrme darıs le Massif Central français, le point des travaux récents. Bulletin de l'Association française pour l'étude du Quaternaire, p. 34-35, 2-3, 103-110.

Goter de Herve, chmus G., Miallier D., Sanzelle S., falgueres C.. Fain J.. Montret M. el Pllleyre T. (1993) - Le puy de Gravenoire et ses coulees, dans l'agglomération de ClemontFerrand (Massif Central français) : un
modèle inhabituel d'avalanche de débris, déclenchée par une éruption strombolienne en climat périglaciaire. Bulletin de la Société géologique de France, 164, n ${ }^{\circ}$ 6, p. 783-793.

Huxtable J., Aitken M.J. et Bonhommet N. (1978) - Thermoluminescence daing of sediments baked by lava flows of the Chaîne des Puys. Nature, 275
p. 207-209.

JUVIGNE E. (1987) - Un marqueur stratigraphique à large dispersion dans le Massif Central français : la retombée du volcan Chopine vieille d'environ 8500 ans B.P., C.R. Acad. Sc. Paris, t. 304 , serie II, $n^{\circ} 4$. p. 187-190.

JuVIGNé E. (1991) - Distribution de vastes retombées volcaniques originaires de l'Eifel et du Massif Central aux temps post-glaciaires dans le NE de la France et les régions voisines. 415-420, 2 fig., 2 tabl.

JUVIGNE $t$. et BASTIN B. (1982) - Découverte d'un tuf volcanique d'âge pré Foreal a la Grande pile (Vosges, France). Bulletin de la Societe geograsept 1982 , p. 31-40.

Juvigne E. et Gewelt M. (1987) - La Narse d'Ampoix comme téphrostratotype dans la Chaîne des Puys méridionale (France). Bulletin de l'Association Irançaise pour l'étude du Quaternaire. 1. p. $37-49$.

JUVIGNÉ E. et G॥OT E. (1986) - Ages et zones de dispersion des téphra mises par les volcans du Montci neyre et du lac Pavin (Massif Central, France). Hannover $Z$. dt. géol. Ges.
137, p. $613-623,2$ fig., 1 tabl.

Juvigné E., KRoonendeng S.B., Welokami A., El Arabi A. el Vernet G. (1992) Widespread Alleröd and Boreal trachyandesitic to trachytic tephra layers as stratigraphical markers in the Massif Quaternaire, 3, (3-4) p. 137-146.

KIeffer G. et Camus G. (1981) - Manifestations dangereuses des volcanls ré putés pacifiques : les éruptions et ex plosions phréatomagmatiques ou phréatiques. Rev. d'Auvergne, t. 95, $n^{\circ} 1$, p. 75-87.

Miallier D. (1982) - L'usage des détecteurs solides de traces dans le cadre de la datation par thermo-lumines cence. Thèse de $3^{\circ}$ cycle, Universite de Ciermont $11, n^{\circ} 711,106 \mathrm{p}$

Miallier D., fain J., Sanzelle S., Daugas J.-P. et RAYNAL J.-P. (1983) - Dating of the Butte de Clermont basaltic maar by means of the quartz inclusion méthod, P.A.C.T. 9, p. 487-498.

Miallier D., Fain J. et Montret M., Pllleyre T., Sanzelle S. et Soumana S. (1991) Properties of the red TL peak of quartz relevant to thermoluminescence dating. Nuclear Tracks and Radiation Measurements, 18 , p. 89-94.

Miallier D., fain J., Sanzelle S, Ray'nal J.P., Daugas J.-P. et Paguereau M.-M. (984) - Datation du volcanisme quaernaire du Massif Central français par la methode des inclusions de quartz en thermoluminescence et comparaison avec d'autres approches. $10^{\circ}$ Réunion annuelle des Sciences de la Terre, Bordeaux, Societé Geologique de France Éd., Paris, p. 396

Marambat L. (1986) - Étude palynologique de la tourbière de la Barthe (Picherande, Puy-de-Dôme). $11^{\circ}$ R.S.T. Clermont-Ferrand, S.G.F. Éd. p. 120 .

Marambat L. (1991) - Étude palynologique de la tourbière de la Barthe (Monts-Dore). In J.-P. Raynal et D. Miallier : Datation et Caracterisation des Milieux Pleistocenes, Cahiers du Quaternaire, C.N.R.S. Ed., $n^{\circ} 16$ p. 181-189. 
MARTINI J. (1970) - Recherches de retombées volcaniques quaternaires dans le S.E. de la France et la Suisse occidentale. Archives des Sciences,
Soc. Phys. Hist. nat. Geneve, vol. 23, p. 641-674.

MAURY R.-C. (1976) - Contamination (par l'encaissant et les enclaves) et cristallsation fractionnée de séries volcaniques alcalines continentales (Massif Central français) et oceaniques (Pacifique Central), l'origine des laves acides. These Doct. Etat, Univ. ParisSud Orsay, 455 p.

MuniheI M., Miallieh D., Sanzelle S., Fain J., Pilleyre T. et Soumanin S. (1992) $\mathrm{TL}$ dating in the Holocene using red $\mathrm{TL}$ from quartz. Ancient TL, vol. 10, $\mathrm{n}^{\circ} 3$ p. 33-36.

Pelletier H. (1964) - Érosion de la vallée de la Monne depuis les dernière eruptions de la chaîne des Puys. Acte du quatre-vingt-huitième congrès national des Sociétés savantes, Clermont-Ferrand. 1963. Imp
tionale, Paris, p. 153-158.

Pellefter H. (1969) - Clermont est -il bât sur un volcan? Alvergne Magazine sur un volcan ? Alwerg
février 1969,18, p. $2-8$.

PILLEYRE T. (1991) - Datation par thermoluminescence Application à la chronologie des retombées volcaniques. These de I'Université Blaise-Pascal, Clermont II, DU 345, $164 \mathrm{p}$.

Pilleyre T., Montret M., Fain J., Miallier D. et SANzelle S. (1991) - Attemps at dating ancient volcanoes using the red TL of quartz. Quaternary Science Review, 11, p. 13-17

RAYNaL J.-P. (1984) - Chronologie des basses terrasses de l'Allier en Grand Limagne (Puy-de-Dôme, France). Bulle$t i n$ de l'Association Française pour
l'Etude du Quaternaire, 1-2-3, p. 79-84.

RAYNAL J.-P. (1987) - Évolution comparée de lacs de maars en Auvergne et en Velay (France) : datation et contribution à la connaissance des climats pléistocènes. Documents du C.E.R.L.A.T., Mémoire $n^{\circ} 1$, p. $65-96$ 10 fig.. 2 tabl.

RaYNAL J.-P. (1988) - Approche du peuplement préhistorique en zone volcanique active, A.T.P. du C.N.R.S. "Approches nouvelles en archéologie", rapport final, 123 p., diffusion restreinte.

RAYNAL J.-P. et DaUGAS J.-P. (1984) Volcanisme et occupation humain dans le Massif Central français quelques observations. Rovuo t. $23,1, \mathrm{p} .7-19$.

Raynal J.-P. et Daugas J.-P. (1989) - Le peuplement paléolithique d'Auvergne.
La Recherche, $n^{\circ} 210$, vol. 20 , p. 690 691.
Raynal J.-P. et Daugas J.-P. (1991) L'homme et les volcans : occupation de l'espace régional à la fin des temps glaciaires dans le Massif Central francais. In Le peuplement magdalénien. Paléogéographie physique et humaine, Actes du Colloque de Chancelade, C.T.H.S. Ed., p. 11-120.

Raynal J.-P. et Huxtable J. (1989) - Premières datations par thermoluminescence du Mousterien charentien du Velay (Massif Central). C.R. Acad. Sci. Paris, t. 309, série II, p. 157-162.

Raynal J.-P. et Sanzelle S. (1989) - Préhistoire en domaine volcanique: exemple de la Basse Auvergne in Le Temps de la Prćhistoirc, Socićtć pró historique française et Archéologia Ed., tome 1, p. 128-129.

Raynal J.-P., Pauvehtau M.-M. et Daugias J.-P. (1981) - Contribution à l'étude chronostratigraphique des formations volcano-sédimentaires de la chaine des Puys. Nouv. Arch. Mus. Hist. nat. Lyon, fasc. 19, p. 59-64, 2 lig.

RAYNAL J.-P.. Daugas J.-P., PaQuereau M.M., Miallier D., Fain J. et Sanzelle S. (1982) - Première datation du maar basallique de Clermonl-Ferrand (Puy-de-Dôme, France) : stratigraphie, palynologie, thermoluminescence. C.A.A.S., Paris, serie II, p. 1011-1014.

Raynal J.-P., Daugas J.-P., Paquereau M.-M., Guadelli J.-L., Marchianti D., Miallier D., Fain J. et SANZELLE $S$. (Puy-de-Dôme, France) : datation par (Puy-de-Dome, France) : datation par thermoluminescence, fores et faunes gossiles, presence humaine, climatololie et dynamique du systeme paleoSc. nat. d'Auvergne, vol. 50, fasc. 12-3-4, Clermont-Ferrand, p. 97-114, 11 fig., 17 tabl.

RaYnal J.-P., Paquereau M.-M., daugas J.-P., MIALLIER D., TaIN J. et SANZELLE S. (1985) - Contribution à la datation du volcanisme quaternaire du Massif Central français par thermoluminescence des inclusions de quartz et comparaison avec d'autres approches : implications chronostratigra Bull. de l'A.F.E.Q., 1985/4, no 24, p. 183207, 10 fig., 4 tabl.

Raynal a.-P., Miali ifr D., Vfrenft G., Fain J., Camus G., Montret M., Daugas J.P. et SANZELLE S. (1989) - Extension de la datation par thermolumines cence à une retombee trachy andósi tique rapportee au Puy de Pariou Scl. Paris, t. 308 , série II, p. 1547-1552.

Verinet G. et Paquereau M.-M. (1986) Le cours moyen de la Morge (Puy-dechronologie et de palécenvironne- ments. $11^{\circ}$ Réunion Annuelle des Clermont-Ferrand, S.G.F. Éd., p. 181

Vernet G., Raynal J.-P., Miallier D. Pilleyre T., Fain J., Sanzelle $S$. e MONTRET M. (1990) - La retombée de la Moutade, marqueur stratlgraphlque de l'Alleröd en Limagne septentrionalc (Massif Central, France). C.R. Acad Sci. Paris, t. 310 , série II, p. 1077 1082.

VLrnet G. et Paqulatau M.-M. (1991) Le cours moyen de la Morge et de ses affluents (Puy-de-Dome. France) au Pléistocène : éléments de chronologie et de paléoenvironnements. In J.-P Raynal et D. Miallier : Datation et caractérisation des milieux pléistocenes, Cahiers du Quaternaire, $n^{n} 16$ C.N.R.S. Éd.

VERNET G. (1991) - Deux niveaux de retombées volcaniques à la base des travertins hydrothermaux de Gimeaux. In : Le monde extraordinaire des sources de Gimeaux, H. Girard Éd. $2^{2}$ trimestre 1991 , p. $7-9,1 \mathrm{fig}$.

Vernet G. (1992) - Message du volcanisme regional dans les formations quaternaires de Limagne occidentale (Massif central français). Minéraux denses et retombées. Thèse de l'Université de Bordeaux 1, $n^{\circ}$ 724, $335 \mathrm{p}$.

Vernet G., raynal J.-P., Fain J., Miallier D. Montret M., Pillifyfa T. et San7flif $S$ (1993) - Tephrostratigraphy of the las $160 \mathrm{ka}$ in Western Limagne (France).. in Quaternary Stratigraphy in Volcanic Areas, Abstracts, Rome, September 1993, p. 61.

VEYRET Y. (1978) - Modelé et formations d'origine glaclaire dans le Massif Central francais. Problcmes de distribution et de limites dans un millieu de moyenne montagne. Thèse de Docto783 p.

Jean-Paul RAYNAL, Gérard VERNET I Iniversité de Bordeaux nstitut du Quaternaire, U.M.R. 9933 C.N.R.S 33405 Talence Cedex Franco.

Jean FAIN, Didier MIALLIER, Michèle MONTRET, Thierry PILLEYRE Serge SANZELLE

Laboratoiro do Physique Corpusculaire 63177 Aubière Cedex Franca Jean-Pierre DAUGAS

Service Rógional de l'Archéologio 1. rue Stanislas-Baudry
44035 Nantes Cedex 01 et U.M.R. 9933 C.N.R.S. 